\title{
Which Women with Vulval High Grade Squamous Intrapethelial Neoplasia (HSIL) should be Considered for Anoscopic Assessments?
}

\author{
Akel Randa, ${ }^{1 *}$ Cohen CE, ${ }^{2}$ Angwin C, ${ }^{1}$ Ume U, ${ }^{2}$ Fuller LC ${ }^{1}$ \\ ${ }^{1}$ Department of Dermatology, Chelsea and Westminster Hospital NHS Foundation Trust, United Kingdom \\ ${ }^{2}$ Department Genitourinary Medicine; Chelsea and Westminster Hospital NHS Foundation Trust, London, United Kingdom
}

\begin{abstract}
Objective: Review the results of anal assessments in women with vulval HSIL with a potential increased risk of anal HSIL, due to HIV status, macroscopic perianal lesions or vaginal/cervical intrepithelial neoplasia.
\end{abstract}

Methods: Ten year retrospective review of 402 anoscopy encounters from 130 patient, who were at higher risk of anal HSIL and underwent anoscopy. Of those, only 13 women had vulval HSIL and were included.

Results: Cohort mean age 51 years, 54\% smoked, 15\% were living with HIV, 31\% had additional vaginal or cervical HPV related pathology and $77 \%$ were referred with macroscopic perianal lesions.

Over half (54\%) had anal biopsies demonstrating HSIL, all of whom had visible pathology.Anal cytology was available for 12 cases, varying from HPV infection (42\%), HG dyskaryosis (25\%) to low grade dyskaryosis (17\%). The entire cohort had either anal HSIL or HPV changes and received treatment: Imiquimod (69\%), laser ablation (54\%), surgical excision (38\%), cryotherapy (31\%), electrocautery (15\%) or Trichloroacetic acid (15\%).

Conclusion: HSIL of the vulva with visible perianal lesions must prompt anoscopy, as anal cytology specificity and sensitivity is inadequate. Vulval services should secure multi-specialty links for screening and treating HPV pathology and neoplasia, which may arise in different anatomical sites.

\section{Introduction}

The pathogenesis of anal cancer appears to resemble that of vulval and cervical cancer.Initial high risk human papilloma virus (HR-HPV) infection could lead to intraepithelial neoplasia and progression from low to high-grade dysplasia, with subsequent invasive cancer potentially arising. ${ }^{1}$ Interestingly the anal transformation zone, which lies above the dentate line, is morphologically analogous to the transformation zone of the cervix. It is the site where most anal intraepithelial neoplasia arises, with highly reactive squamous cells reacting to $\mathrm{HR}-\mathrm{HPV}^{2}$ There is evidence to suggest that women with cervical HR-HPV are at increased risk of anal HR-HPV, mainly HPV 16 \& HPV 18. This implies that anal infec tion with HPV could be secondarily acquired following initial cervi cal infection, possibly due to their anatomical proximity. ${ }^{3}$ However, anal cancer is fairly rare and the risk of being diagnosed with anal cancer is approximately 1 in 500 adults, although the incidence is increasing in both men and women.Over the past three decades the incidence of anal cancer in women has doubled, not as a result of increasing screening but due to a multitude of other factors. ${ }^{4}$ Common risk factors include a history of HPV infection, cigarette smoking, receptive anal sex and lifetime number of sexual partners. ${ }^{5} \mathrm{Ad}-$ ditional groups identified to have an increased risk include solid organ transplant recipients and individuals living with HIV. Also at increased risk are women with a history of cancer or HSIL of the cervix and/or vulva. ${ }^{3,6,7}$ Consideration should therefore be given to
Quick Response Code:

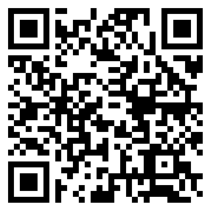

*Corresponding author: AkelRanda, Department of Dermatology, Chelsea and Westminster Hospital NHS Foundation Trust, United Kingdom

Received: 18 January, 2021

Published: 04 March, 2021

Citation: Akel Randa, Cohen CE, Angwin C, Ume U, Fuller LC. Which Women with Vulval High Grade Squamous Intrapethelial Neoplasia (HSIL) should be Considered for Anoscopic Assessments?. Derma Cosm Int J. 2021;1(1):1-3.DOI: 10.53902/DCIJ.2021.01.000502 
anal screening and early intervention, similar to cervical screening, within these groups.

\section{Report}

Our study aimed to review the results of anal assessments in women with vulval HSIL with a potential increased risk of anal HSIL, due to HIV status, macroscopic perianal lesions or vaginal/cervical intrepithelial neoplasia. We conducted a retrospective review of all female patients seen across our two hospital sites in the last 10 years who underwent anoscopic assessment. Out of 402 anoscopic encounters, only 13 patients were found to have HSIL of the vulva (Figure 1). Medical records were reviewed to include the patients' age at the time of anoscopy, HIV and smoking status, correlation with clinical findings, results of the anal biopsy, anal cytology and inclusion of subsequent management. The mean age of the vulvar HSIL cohort at anoscopy was 51 years, ranging from 32 to 72 , with 77\% (10) European Caucasian ethnicity, 15\% (2) Black African and 8\% (1) Latin American.Over half were smokers (54\%, 7) but only 15\% (2) were living with HIV.Thirty one per cent (4) had additional HPV related pathology (VaIN, CIN, vaginal squamous cell carcinoma). Notably, the majority of these women were referred because of macroscopic perianal lesions $(77 \%, 10)$.Over half of the referred cases had an anal biopsy, which demonstrated HSIL of the anus $(54 \%, 7)$ and all of these patients had visible perianal pathology. The majority of biopsy sample $(86 \%, 6)$ were from HIV negative patients and just under half $(43 \%, 3)$, had another focus of HPV related neoplasia (CIN I, CIN II, vaginal squamous cell carcinoma).Anal cytology was available for 12 of the 13 cases.Results varied from HPV infection $(42 \%, 5)$, high grade dyskaryosis $(25 \%, 3)$, low grade dyskaryosis $(17 \%, 2)$ and insufficient sample $(17 \%, 2)$. All patients who underwent anoscopy received treatment for either either HSIL of the anus or other HPV related changes (Figure 2).

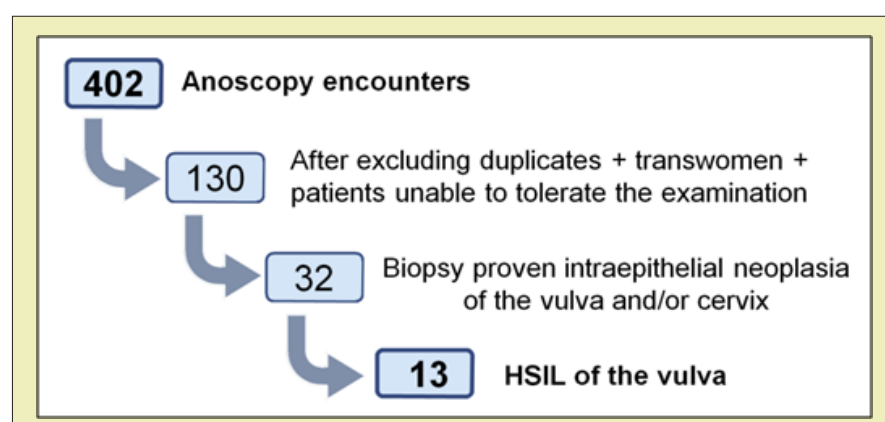

Figure 1: Data process flow chart.

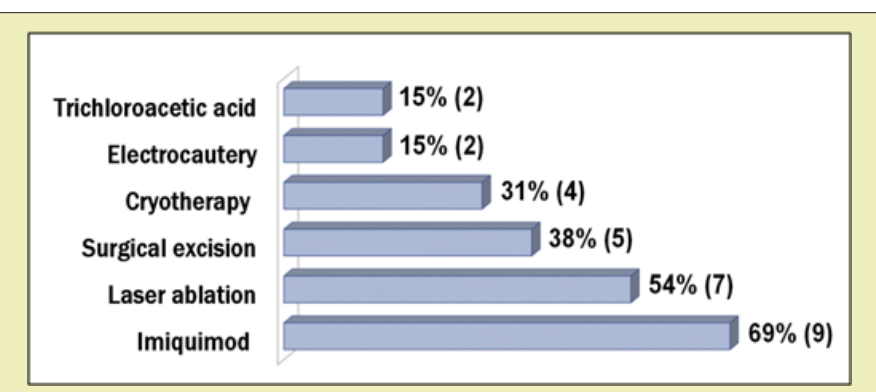

Figure 2: Treatment provided following high resolution anoscopy.

\section{Discussion}

High resolution anoscopy remains the gold standard for anal cancer screening, but requires specialist expertise, equipment and facilities. Anal cytology has been suggested as a cost effective and sensitive alternative screening tool in different cohorts, particularly HIV positive men-who-have-sex-with-men, ${ }^{8}$ as it is more widely available, time efficient and less invasive for patients. Interestingly, our results did not find concordance between anal cytology and biopsy in relation to the degree of dysplasia. This concurs with literature reviews which have highlighted that the estimated sensitivity and specificity of anal cytology can vary widely, with the sensitivity decreasing in the highest risk groups, making the specificity for diagnosing the correct degree of dysplasia even less accurate.Accordingly, some specialist centres have developed stratified screening algorithms for their at-risk groups, incorporating these limitations. ${ }^{9}$ What remains clear is that the optimal anal screening strategy for women with vulval HSIL with or without additional risk factors, including HIV, is controversial and guidelines in this area are lacking. There are no randomised control studies that have demonstrated the advantage or cost effectiveness of routinely screening in these high-risk female populations. We suggest HSIL of the vulva with visible perianal lesions should prompt high resolution anoscopy, as anal cytology specificity and sensitivity remain inadequate. Vulval services should secure multi-specialty links for screening and treating HPV pathology and neoplasia, which may arise in different anatomical sites. The value of these co-ordinated resources should not be underestimated or overlooked despite the impact of the current Covid-19 pandemic, in order to prevent delays in detectable and treatable disease.

\section{Learning points}

I. Review of the perianal area must form part of a vulval clinical examination.

II. HSIL of the vulva with visible perianal lesions must prompt high resolution anoscopy.

III. Anal cytology and specificity can be inadequate to determine HSIL of the anus.

IV. Vulval services must secure multi-specialty links for screening and treating HPV related pathology.

\section{Acknowledgments}

None.

\section{Conflicts of interest}

None.

\section{Funding}

None.

\section{References}

1. Allice Hocking, Adam Fisher, Lorna Bell. Screening for Anal Cancer. External review against programme appraisal criteria for the UK National Screening Committee (UK NSC). 2012. 
2. Cronin, Amy Bregar, Christine Luis, et al. Evaluation of anal cytology and dysplasia in women with a history of lower genital tract dysplasia and malignancy. Gynecol Oncol. 2016;141(3):492-496.

3. Stanley MA, Winder DM, Sterling JC, et al. HPV infection, anal intraepithelial neoplasia (AIN) and anal cancer: current issues. BMC cancer. 2012;12:398.

4. Shiels MS, Kreimer AR, Coghill AE. Anal Cancer Incidence in the United States, 1977-2011: Distinct Patterns by Histology and Behavior. Cancer epidemiology, biomarkers \& prevention: a publication of the American Association for Cancer Research, cosponsored by the American Society of Preventive Oncology. 2015;24(10):1548-1556.

5. Nyitray Alan, Lu Beibei, KreimerAimée. The Epidemiology and Control of Human Papillomavirus Infection and Clinical Disease. Sexually Transmitted Diseases. 2013;315-352.
6. Santoso Joseph T, Long Mary, Crigger Mary, et al. Anal Intraepithelial Neoplasia in Women with Genital Intraepithelial Neoplasia. Obstetrics \& Gynecology. 2010;116(3):578-582.

7. Joël FokomDomgue, Craig Messick, Andrea Milbourne, et al. Prevalence of high-grade anal dysplasia among women with high-grade lower genital tract dysplasia or cancer: Results of a pilot study. Gynecologic Oncology. 2019;153(2):266-270.

8. Gaisa M, Goldstone E. Diagnosis and treatment of anal intraepithelial neoplasia and condylomata. Seminars inColon \& Rectal Surgery. 2011;22:21-30.

9. Morency EG, Harbert T, Fatima N, et al. Anal Cytology: Institutional Statistics, Correlation With Histology, and Development of Multidisciplinary Screening Program With Review of the Current Literature. Arch Pathol Lab Med. 2019;143(1):23-29. 